\title{
COMPOSITION AND SPECTRAL PROPERTIES OF THE 1 AU QUIET-TIME SUPRATHERMAL ION POPULATION DURING SOLAR CYCLE 23
}

\author{
M. A. Dayeh ${ }^{1}$, M. I. Desai ${ }^{1}$, J. R. Dwyer ${ }^{2}$, H. K. Rassoul ${ }^{2}$, G. M. Mason ${ }^{3}$, and J. E. Mazur ${ }^{4}$ \\ ${ }^{1}$ Southwest Research Institute, San Antonio, TX 78238, USA; maldayeh@swri.edu \\ ${ }^{2}$ Florida Institute of Technology, Melbourne, FL 32901, USA \\ ${ }^{3}$ The Johns Hopkins University Applied Physics Laboratory, Laurel, MD 20723, USA \\ ${ }^{4}$ The Aerospace Corporation, Chantilly, VA 20151, USA \\ Received 2008 September 10; accepted 2008 December 2; published 2009 March 10
}

\begin{abstract}
We have surveyed the spectral and compositional properties of suprathermal heavy ions during quiet times from 1995 January 1 to 2007 December 31 using Wind/Energetic Particles: Anisotropy, Composition, and Transport/SupraThermal-through-Energetic Particle Telescope and Advanced Composition Explorer/Ultra-low Energy Isotope Spectrometer at energies between 0.04 and $2.56 \mathrm{MeV}$ nucleon $^{-1}$. We find the following. (1) Quiettime $\mathrm{Fe} / \mathrm{O}$ and $\mathrm{C} / \mathrm{O}$ abundances are correlated with solar cycle activity, reflecting corresponding values measured in solar energetic particle and interplanetary (IP) shock events during solar maximum, and those measured in the solar wind and corotating interaction regions (CIRs) during solar minimum conditions. (2) The ${ }^{3} \mathrm{He} /{ }^{4} \mathrm{He}$ ratio lies in the 3\%-8\% range during the quiet times of 1998-2004 with finite ${ }^{3} \mathrm{He}$ detected on $\sim 27.4 \%$ of the days. This ratio drops to $0.3 \%-1.2 \%$ during $2005-2007$ and finite ${ }^{3} \mathrm{He}$ is detected on $\sim 5 \%$ of the days. (3) All heavy-ion species exhibit suprathermal tails between 0.04 and $0.32 \mathrm{MeV}^{-1}$ ncleon $^{-1}$ with spectral indices ranging from $\sim 1.27$ to 2.29. These tails sometimes extend above $\sim 2 \mathrm{MeV}$ nucleon $^{-1}$ with Fe spectra rolling over at lower energies than those of CNO. (4) The suprathermal tail spectral indices of heavier species (i.e., Fe) are harder than those of the lighter ones (i.e., CNO). These indices do not exhibit a clear solar cycle dependence and for $\sim 50 \%$ of the time, they deviate significantly from the 1.5 value. These compositional observations provide evidence that even during the quietest times in IP space, the suprathermal population $\left({ }^{3} \mathrm{He}\right.$ and $\mathrm{C}$-through-Fe) consists of ions from different sources whose relative contributions vary with solar activity. The heavy-ion energy spectra exhibit suprathermal tails with variable spectral indices that do not exhibit the spectral index of 1.5 predicted by some recent models.
\end{abstract}

Key words: acceleration of particles - interplanetary medium - Sun: abundances

\section{INTRODUCTION}

Recent measurements from mass spectrometers on board Wind (Acuña et al. 1995) and the Advanced Composition Explorer (ACE; Stone et al. 1998) spacecraft have shown that a sizable fraction of CME-driven interplanetary (IP) shocks (Desai et al. 2001), large solar energetic particle (LSEP) events (Mason et al. 1999; Cohen et al. 2000; Desai et al. 2006b), and particle events associated with corotating interaction regions (CIRs) (Chotoo et al. 2000; Kucharek et al. 2003; Mason et al. 2008) exhibit substantial enrichments in the abundances of ions such as $\mathrm{He}^{+}$and ${ }^{3} \mathrm{He}$ which are very rare in the solar wind (SW; Gloeckler \& Geiss 2007). These ion species serve as tracers of their origin; the ${ }^{3} \mathrm{He}$ ions originate from solar flares (e.g., Mason et al. 2002) while the $\mathrm{He}^{+}$ions are interstellar neutral atoms that penetrate into the inner heliosphere inside $1 \mathrm{AU}$, and then get ionized and "picked-up" by the out-flowing SW (Möbius et al. 1985; Gloeckler et al. 1994). In addition, detailed studies have also shown that the abundances of heavy ions from C-Fe in IP shock-related and in solar energetic particle (SEP) events are not organized by a single physical quantity such as mass-per-charge (M/Q) or first ionization potential (FIP) (Mewaldt et al. 2007; Desai et al. 2003, 2006a). The mere presence of these tracer ion species in the SEP and CIR-accelerated populations, taken together with the lack of correlations between SEP heavy-ion abundances and those measured in the SW provide compelling evidence that the particles accelerated by CIR and CME-driven shocks do not originate primarily from the bulk SW, but rather from a hotter $(\sim 1.5-2$ times that of the bulk SW) and more complex unexplored regime (e.g., Mason et al., 1999). Thus, in order to fully understand the physical processes such as injection, acceleration, and IP transport of SEPs and IP-shockrelated events it is critical that we first identify the origin of the seed particles and characterize their properties.

Desai et al. (2006a) found that the suprathermal heavy population near 1 AU during quiet times from 1994 through 2006 is largely dominated by SEP material during solar maximum and by CIR or SW-like material during solar minimum. This indicates that the suprathermal population is highly dynamic and includes ions accelerated during prior solar and IP activities (Mason et al. 1999; Mason 2000), in addition to interstellar and inner-source pickup ions, and heated SW ions (Gloeckler et al. 1994, 2000; Mason et al. 2008).

Moreover, recent work has shown that during different plasma conditions in IP space, the suprathermal tails, also known as quiet-time tails, exhibit a unique power law of spectral index $E^{-1.5}$ when expressed as a function of differential intensity in energy $E$, or $v^{-5}$ when expressed as a distribution function in velocity space (Simunac \& Armstrong 2004; Fisk \& Gloeckler 2006, 2007a, 2007b; Gloeckler et al. 2008). These ubiquitous power-law tails, observed in both protons and heavy ions, appear to be dominated by SW ions and CIRs at $1 \mathrm{AU}$, and interstellar pickup ions beyond 1 AU (see also Gloeckler 2003). Interestingly, these results could not be explained by two of the most widely accepted ion acceleration processes in space, namely: (1) stochastic acceleration in the SW (e.g., Fisk 1976; Schwadron et al. 1996; le Roux et al. 2001, 2002; Giacalone et al. 2002; Webb et al. 2003), and (2) diffusive shock acceleration, also known as first-order Fermi acceleration (e.g., Blandford \& Ostriker 1978; Bell 1978). Based on these observations, Fisk \& Gloeckler (2006, 2007a) suggested that there 

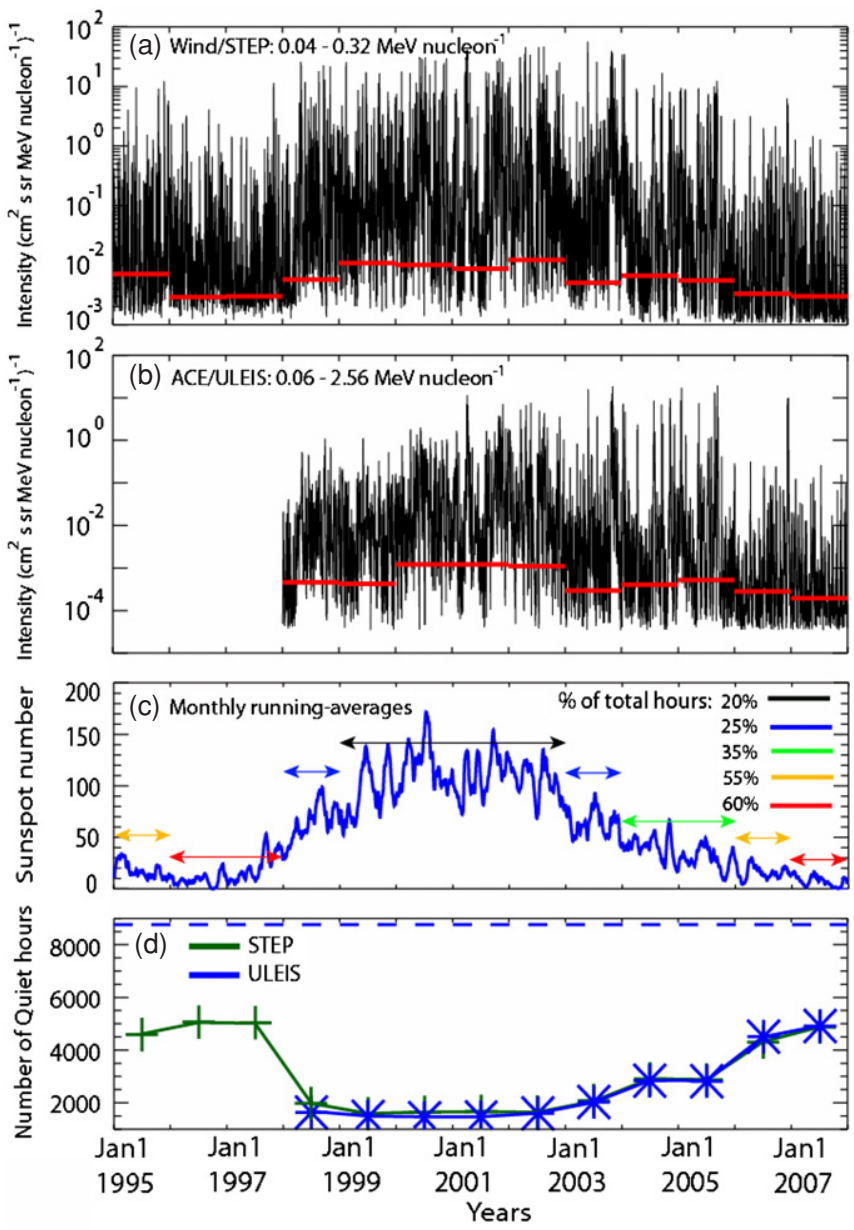

Figure 1. Six-hour running averages of heavy ions ( $\mathrm{C}$ through $\mathrm{Fe}$ ) intensity as measured by ( $a$ ) ACE/ULEIS and (b) Wind/STEP at two different energy ranges; red bars mark the maximum intensity of the quiet times during each year. (c) Monthly running averages of sunspot numbers from 1995 January 1 through 2007 December 31. Assigned percentages denote the fraction of total hours that are considered quiet. $(d)$ Number of quiet hours during each year as a result of the selection criteria in ULEIS (blue) and STEP (green). The dashed blue line marks the total number of hours in a year.

is an additional acceleration process that yields unique spectra under different plasma conditions and at distinct locations in the heliosphere, ranging from planetary magnetosheaths to the heliosheath beyond the SW termination shock (see Stone et al. 2005). They introduced a theory based on thermodynamic constraints suggesting that these unique spectral tails can be produced by stochastic acceleration due to compressional turbulence in the plasma, when no shocks are present.

One approach for characterizing the suprathermal population is to study the properties of the material present during the quietest conditions in the IP medium. During such periods, the quiet IP medium is essentially devoid of ions associated with transient solar activity or with quasi-steady IP disturbances like CIRs. Thus, particle populations during carefully selected quiet times can be assumed to best describe the suprathermal pool of material present in the IP medium that may be available for acceleration at CIR and CME-driven IP shocks. In this paper, we examine the composition and spectral properties of suprathermal ions ( ${ }^{3} \mathrm{He}$, and C-through-Fe) from 1995 January 1 to 2007 December 31 during such quiet times at energies between $\sim 0.04$ and 2.56 MeV nucleon ${ }^{-1}$ using Wind/SupraThermal-throughEnergetic Particle Telescope (STEP) and Advanced Composition Explorer/Ultra-low Energy Isotope Spectrometer (ACE)
ULEIS). We find that the quiet-time suprathermal population $\left({ }^{3} \mathrm{He}\right.$ and $\left.\mathrm{C}-\mathrm{Fe}\right)$ is largely affected by the solar cycle activity, with SEP-like composition signatures during solar maximum and CIR or SW-like composition during solar minimum. In addition, we find that during quietest times in the IP medium, all ions exhibit suprathermal tails with spectral indices ranging from 1.27 to 2.29 , significantly different from recent reported observations and theoretical predictions of a unique 1.5 index (see Fisk \& Gloekler 2006, 2007a, 2007b; Gloeckler et al. 2008). We discuss these new observations and their implications for the origin of the suprathermal ion population in IP space near $1 \mathrm{AU}$.

\section{INSTRUMENTATION}

Data analyzed in this paper are obtained from two instruments onboard two spacecraft (1) the STEP instrument within the Energetic Particles, Acceleration, Composition, and Transport Experiment (EPACT; von Rosenvinge et al. 1995) onboard Wind, which was launched in 1994 November. STEP is a timeof-flight (TOF) versus residual energy mass spectrometer that resolves elements from $\mathrm{H}$ to $\mathrm{Ni}$ for energies between $\sim 0.02$ $\mathrm{MeV}$ nucleon $^{-1}$ and $\sim 2 \mathrm{MeV}$ nucleon $^{-1}$. It has a geometry factor of $0.4 \mathrm{~cm}^{2} \mathrm{sr}$ and a $10 \mathrm{~cm}$ TOF path. (2) The ULEIS (Mason et al. 1998) on board ACE, launched in 1997 August, is also a TOF versus residual energy mass spectrometer with a flight path of $50 \mathrm{~cm}$ and a geometry factor of $\sim 1 \mathrm{~cm}^{2} \mathrm{sr}$. ULEIS resolves $\mathrm{H}-\mathrm{Ni}$ ions between $\sim 0.045 \mathrm{MeV}$ nucleon $^{-1}$ and $\sim 10 \mathrm{MeV}^{\text {nucleon }}{ }^{-1}$. STEP and ULEIS have higher sensitivity with low background rates, larger geometry factors, and better mass resolution than previously flown mass spectrometers in similar energy ranges. Wind/STEP provides ion measurements over the complete solar cycle 23 and both Wind STEP and $A C E /$ ULEIS provide simultaneous data for in situ suprathermal through energetic particle measurements at $1 \mathrm{AU}$ as of 1997 September.

\section{SELECTION OF QUIET PERIODS}

\subsection{Selection Criteria}

Selecting quiet times over an entire solar cycle for different species at different energy ranges is somewhat subjective, mainly due to the fact that there is no constant, "quiet" rate which sets a floor on intensity levels. In selecting the quiet times for our study, we first derived the heavy-ion ( $\mathrm{C}$ through $\mathrm{Fe}$ ) hourly averaged intensity (heavy-ion intensity hereafter) over 0.04 $0.32 \mathrm{MeV}$ nucleon $^{-1}$ for STEP, and 0.06-2.56 $\mathrm{MeV}$ nucleon $^{-1}$ for ULEIS. We use hourly averages instead of daily averages (as in Desai et al. 2006a) to select the quiet times because it has allowed us to exclude multi-hour events (e.g., upstream events from the Earth's bow shock), short-time saturation periods on both spacecraft, and energy-specific enhancements (e.g., velocity dispersion during SEP events, see Mazur et al. 2000). For each hour, this intensity represents all detected heavy ions ( $\mathrm{C}$ through $\mathrm{Fe}$ ) that fall within the selected energy range. For each year, we then created an intensity-hour histogram and defined the quiet times to be a certain variable fraction (between $\sim 20 \%$ and $60 \%$ ) of the hours that represent the lowest values of the heavy-ion intensity. This percentage difference is necessary because the number of hours with zero counts during solar minimum years is much higher than that during solar maximum years. We emphasize that our selected quiet times represent a fraction of the total hours with the lowest values for the hourly averaged heavy ion intensity over a wide energy range in a 
given year and should not be taken as indicative of an intensity threshold level for a certain species in a narrow energy range.

Figure 1 provides an overview of the results of our selection criteria. Figures 1 (a) \& (b) show the heavy-ion intensity over the selected energy ranges from STEP and ULEIS, respectively, along with the highest quiet-times intensity levels (red bars) for each year. Note that although our selection criteria yielded different intensity levels for different years, the maximum intensity level for all years lies within an order of magnitude on both instruments.

Figure 1(c) shows the percentage values used in selecting the quiet hours for different years along with the 27 day running averages of sunspot numbers. In particular, we defined the quiet times to be $20 \%$ of the hours with the lowest values of the heavy-ion intensity during 1999-2002, 25\% during 1998, 2003 ; $35 \%$ in 2004, 2005; 55\% during 1995, 2006; and 60\% during 1996, 1997 (for STEP), and 2007. During solar minimum years, we considered those hours representing the lowest $60 \%$ of the intensity (versus $20 \%$ during solar maximum) because the heavy-ion intensity during these years is mostly due to single counts from a single ion species in a narrow energy range, implying that all other species at other energies have zero counts, and thus resulting in a much lower heavy-ion intensity level. Note that although the fraction of quiet hours during solar minimum is 3 times that during solar maximum years, the highest quiet-time intensity during solar minimum is still lower than that during solar maximum years (red bars).

Figure 1(d) shows that our selection criteria resulted in different numbers of quiet hours for different years. We used these quiet hours to derive the ${ }^{3} \mathrm{He}$ abundances and the composition and spectral properties of C-Fe ions over solar cycle 23. We remark that our selection technique resulted in remarkable agreement between the quiet-time energy spectra from STEP and ULEIS at overlapping energies, thereby allowing us to extend the energy range of suprathermal heavy-ion spectra. In order to test the robustness of our selection criteria, we also compared our results with those obtained using different selection criteria for both data sets. For instance, we selected quiet times on the basis of fixed intensity levels for individual species (He, $\mathrm{CNO}$, and $\mathrm{Fe}$ ) in different energy ranges. However, we found that these alternative selection criteria were not successful in eliminating periods with energy-specific enhancements (e.g., upstream events at low energies and high-energy enhancements due to velocity dispersion during SEP onsets), which is critical for deriving the spectral properties of the quiet-time heavy-ion suprathermal population over nearly two decades in energy.

\subsection{Examples of Quiet Hours}

Figures 2 and 3 investigate the occurrence of the quiet times and the robustness of the selection criteria during 2 years representative of solar maximum (2001) and solar minimum (2007), respectively. Figure 2(a) shows a histogram of the heavy-ion intensity along with the level corresponding to those hours that had the lowest $20 \%$ intensities. Figure 2(b) shows a histogram distribution of all consecutive quiet hours during 2001; the highest being 78 consecutive hours between day-ofyear (DOY) 36 and 40. Figures 2(c)-(g) illustrate the selection criteria on different species in narrower energy ranges. Figure 2(c) shows the hourly averaged heavy-ion intensity (black dots) and during quiet times (red dots in the yellowshaded regions) based on the $20 \%$ level previously described. Figures 2(d)-(g) show the $\mathrm{CNO}$ and $\mathrm{Fe}$ intensities during nonquiet (black) and quiet (red) times in two different energy ranges.
Note that SEP enhancements prior to DOY 34 and after DOY 43, 15:00 UT are totally excluded, and our selected quiet times represent times with the lowest intensity levels for all species in all energy ranges shown. Also, note the gaps interrupting the consecutivity of quiet-time stretches (e.g., between yellowshaded regions A and E in Figure 2(d)). During these shortlived gaps, only the CNO intensity in the lowest energy range (Figure 2(d)) is enhanced, while other intensities remain low (e.g., Figures 2(e) $-(\mathrm{g}))$. The $\mathrm{CNO}$ enhancement during these gaps caused the average heavy-ion intensity (in Figure 2(c)) to increase above our selection threshold, so the periods were excluded.

Our selection criteria also eliminates hours with intensity enhancements that occur only at higher energies due to velocity dispersion effects that typically occur during the onsets of SEP events (Mason et al. 1999, Desai et al. 2006b). For example, in Figure $2(\mathrm{~g})$ the Fe intensity between DOY 33 and 34 at 0.16 $0.32 \mathrm{MeV}$ nucleon ${ }^{-1}$ (green shaded box) is low, while during the same period, the CNO intensity at $0.04-0.08 \mathrm{MeV}^{\text {nucleon }}{ }^{-1}$ (Figure 2(d)) shows the decay of an SEP event. This also enhances the heavy-ion intensity in figure 2(c) above the cutoff threshold. Figure 3 shows a similar plot to that of Figure 2 but for 2007. Figure 3(b) shows that the largest number of consecutive quiet hours during 2007 is $\sim 130$, higher than that during 2001 . Although SEP enhancements are less frequent during this year and the number of consecutive hours is significantly higher, the quiet times are often interrupted by small enhancements at the lowest energies as shown in Figure 3(c) (green shaded box), most likely from upstream events (see Dwyer et al. 2000; Desai et al. 2008).

Figure 4 shows the cumulative heavy-ion intensity during the quiet hours of 2001 and 2007. The smooth increase of the cumulative intensity during each year implies that our selected quiet times are not dominated by a large increase in the count rate within a relatively short period (few hours) compared with the total number of quiet hours that are used to derive the composition and spectra. We finally remark that (see Figures 2 and 3) our selection criteria has ensured that in any given hour, the maximum count rate used to derive the heavy-ion intensity did not exceed seven counts, while the average count rate for any given species in narrower energy ranges did not exceed the three count level (see Figures 2 and 3, panels d-g).

\section{RESULTS}

\subsection{Suprathermal Compositional Variations During the Quiet Times}

Using STEP and ULEIS data during the quiet times described above, we calculated the elemental abundances of heavy ions $\left(\mathrm{C}\right.$ through $\mathrm{Fe}$ ) and those of ${ }^{3} \mathrm{He} /{ }^{4} \mathrm{He}$ in the energy range 0.32 $0.64 \mathrm{MeV}$ nucleon ${ }^{-1}$. We then compared these abundances with previous results obtained using different selection criteria for the quiet times (Desai et al. 2006a defined quiet times where the $\sim 0.12 \mathrm{MeV}$ nucleon ${ }^{-1} \mathrm{Fe}$ intensity was less than $10^{-4}$ particles $\left.\left(\mathrm{cm}^{2} \mathrm{~s} \mathrm{sr} \mathrm{MeV} \mathrm{nucleon}{ }^{-1}\right)^{-1}\right)$. Table 1 lists the annual-averaged elemental abundances of $0.32-0.64 \mathrm{MeV}$ nucleon ${ }^{-1}$ of ${ }^{3} \mathrm{He} /{ }^{4} \mathrm{He}$ and $\mathrm{C}$ through $\mathrm{Fe}$ during the quiet times of solar cycle 23. For all species, an upper limit is given when the statistical uncertainties exceeded $35 \%$.

\subsubsection{Heavy-Ion Composition}

Figure 5(a) shows the yearly ULEIS 0.32-0.64 MeV nucleon ${ }^{-1} \mathrm{C} / \mathrm{O}$ ratio and that calculated by Desai et al. (2006a) 

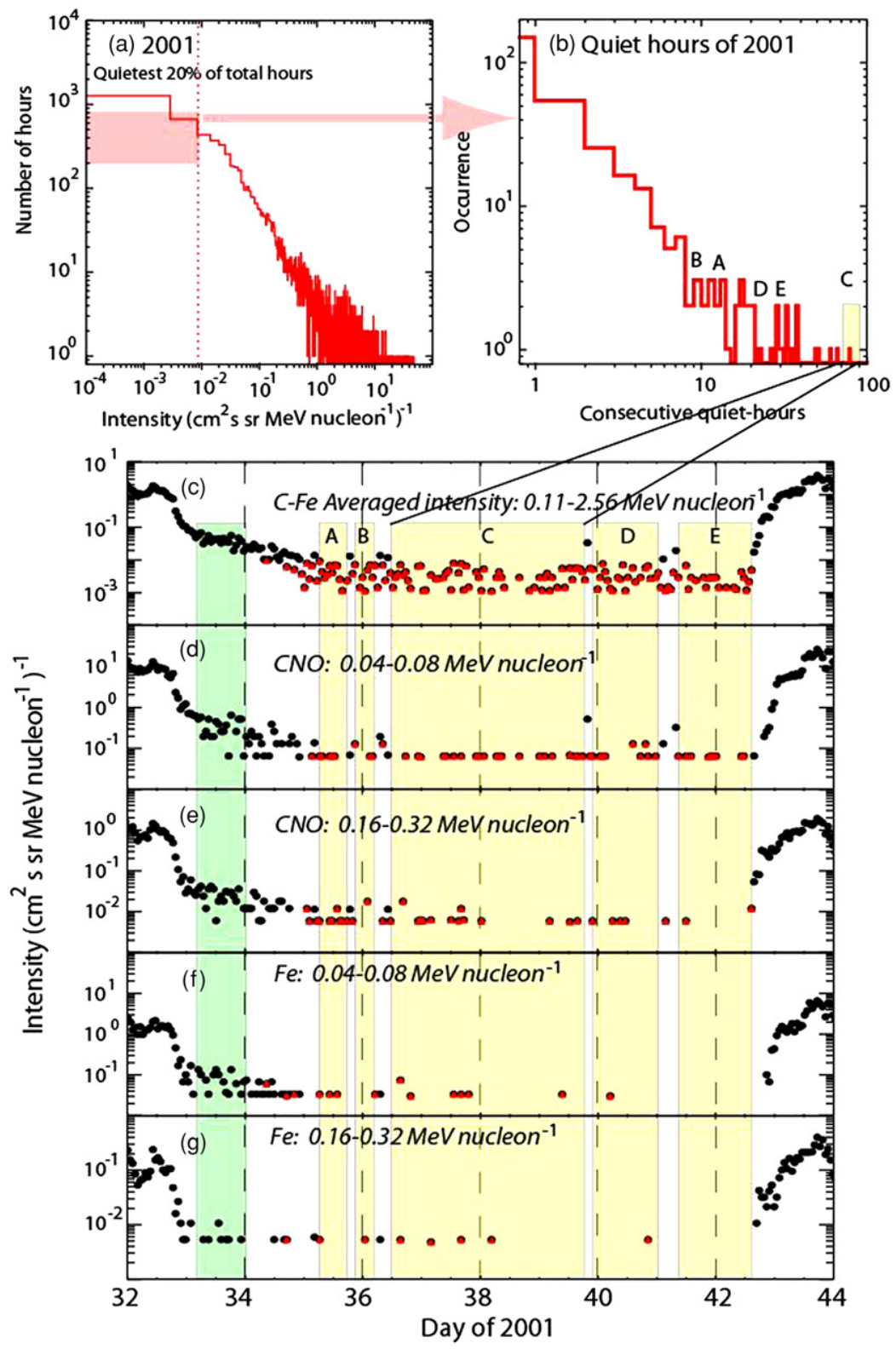

Figure 2. (a) Heavy-ion intensity histogram for all hours as measured by STEP during 2001. The dashed line shows the highest heavy-ion intensity cutoff during the selected quiet times. (b) Distribution of the consecutive quiet hours. ( $c$ ) $-(g)$ Hourly intensities of different species at different energy ranges (black). The red symbols indicate the quiet-time intensities. The yellow-highlighted regions mark intensities with consecutive quiet hours corresponding to their different occurrences in $(b)$. The green shaded areas are discussed in the text.

at $0.08-0.16 \mathrm{MeV}$ nucleon $^{-1}$ using STEP. Figure 5(b) shows the $\mathrm{Fe} / \mathrm{O}$ ratio from ULEIS and STEP Fe/CNO (this work) and $\mathrm{Fe} / \mathrm{O}$ (Desai et al. 2006a) at similar energies as in Figure 5(a). For comparison purposes, horizontal lines in Figures 5(a) and (b) indicate the averaged abundances of $\mathrm{C} / \mathrm{O}$ and $\mathrm{Fe} / \mathrm{O}$ measured in ${ }^{3} \mathrm{He}$-rich (Mason et al. 2004) and large gradual SEP (Desai et al. 2006a) events, CME-driven IP shock events (Desai et al. 2003), fast SW (Gloeckler \& Geiss 2007), slow SW (von Steiger et al. 2000), and CIRs (Mason et al. 2008).

Figure 5(a) shows that during 1998-2006, the C/O ratio follows a trend similar to that shown by Desai et al. (2006a), reflecting SEP-like values during years of solar maximum, and SW and CIR-like material during solar minimum with overall variation by a factor of $\sim 1.5$. The $\mathrm{Fe} / \mathrm{O}$ ratio in Figure 5 (b) shows a strong dependence on the solar cycle activity varying by more than a factor of $\sim 4$, with large enhancements during solar maximum years (e.g., 2000 and 2001), and significantly lower values during solar minimum. The figure shows that the quiet- time $\mathrm{Fe} / \mathrm{CNO}$ ratio varies by more than an order of magnitude between 2001 and 2007 (blue).

\subsection{2. ${ }^{3} \mathrm{He}$ Abundance}

Figure 5(c) shows the $0.32-0.64 \mathrm{MeV}$ nucleon ${ }^{-1}{ }^{3} \mathrm{He} /{ }^{4} \mathrm{He}$ ratio measured by ULEIS compared with that measured by Desai et al. at $0.35-1 \mathrm{MeV}$ nucleon $^{-1}$ along with the SWaveraged value (Gloeckler \& Geiss 1998). The ${ }^{3} \mathrm{He} /{ }^{4} \mathrm{He}$ ratio is calculated by dividing the total number of counts of ${ }^{3} \mathrm{He}$ by those of ${ }^{4} \mathrm{He}$ during the quiet times for each year. ${ }^{3} \mathrm{He}$ ions were taken as those with mass between 2.65 and 3.2 amu and above the extrapolated tail of the ${ }^{4} \mathrm{He}$ distribution. The ${ }^{4} \mathrm{He}$ background is subtracted from the ${ }^{3} \mathrm{He}$ mass histogram. We remark that for all the years, the ${ }^{3} \mathrm{He}$ peak was well resolved (e.g., see Desai et al. 2006a).

In this figure, the annual-averaged ${ }^{3} \mathrm{He} /{ }^{4} \mathrm{He}$ ratio during the quiet times lies between 3\% and 7.8\% during 1998-2007 with 


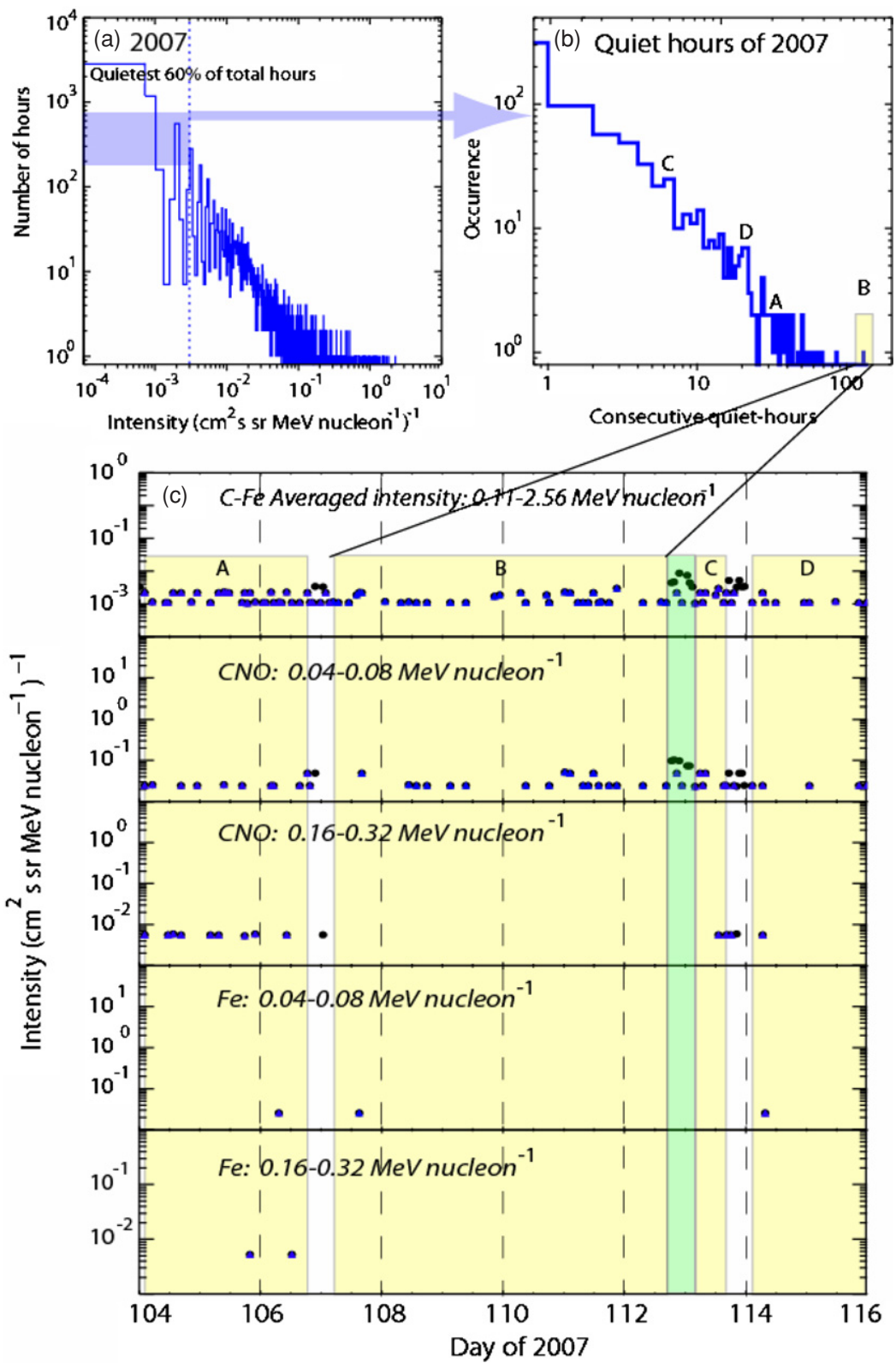

Figure 3. Same as Figure 2, but for 2007.

an average of $\sim 6 \%$. The ratio then drops by more than an order magnitude during 2005-2007 and ranges between 0.3\% and $1.2 \%$ with an average of $\sim 0.7 \%$. However, both values are significantly larger than the averaged abundance measured in the SW $(\sim 0.04 \%)$. We note that even though the average ${ }^{3} \mathrm{He} /$ ${ }^{4} \mathrm{He}$ ratio follows the same trend as that reported by Desai et al. the values obtained in this work are somewhat higher, reaching a factor of $\sim 5$ in some years. This discrepancy is likely because both studies did not consider ${ }^{3} \mathrm{He}$ or ${ }^{4} \mathrm{He}$ in their selection of the quiet times and could be including different multiday ${ }^{3} \mathrm{He}$-rich periods and are not associated with resolved ${ }^{3} \mathrm{He}$ injections (see Kocharov et al. 2008).

To investigate the presence of ${ }^{3} \mathrm{He}$ during the quiet times, we show in Figure 6, the ${ }^{3} \mathrm{He}$ abundance in two Bartel's rotations representing 2 years with different solar activity levels (2000 and 2007). Figure 6(a) shows the average heavy-ion intensity for Bartel's rotation number 2275 (days 76-103 of 2000). All quiet hours, represented by the red dots, are associated with the lowest heavy-ion intensity and tend to occur between different SEP events during solar maximum. This is typical of all quiet hours identified during periods with high solar activity levels (1998-2003). The arrival of ${ }^{3} \mathrm{He}$ ions during these quiet hours is shown in Figure 6(c). Figures 6(b) and (d) show similar plots but for Bartels rotation number 2367 (days 3-30 of 2007). We note that although the actual number of quiet hours is significantly larger during the 2367 (in 2007) Bartels rotation period, ${ }^{3} \mathrm{He}$ is present only $\sim 5 \%$ of the time (10 out of 214 quiet hours), while it is detectable $93 \%$ of the time (26 out of 28 quiet hours) during the quiet hours of the 2275 (in 2001) Bartel's rotation.

Previous studies by Wiedenbeck et al. $(2003,2005)$ found that ${ }^{3} \mathrm{He}$ (at energies greater than $4.5 \mathrm{MeV}$ nucleon ${ }^{-1}$ ) is present at least $60 \%$ of all times in the IP space during different years of the solar cycle. To investigate the presence of ${ }^{3} \mathrm{He}$ during the quiet hours over different levels of solar activity, we repeated the analysis of Figure 6 for all Bartels rotations from 1998 to 2007. 


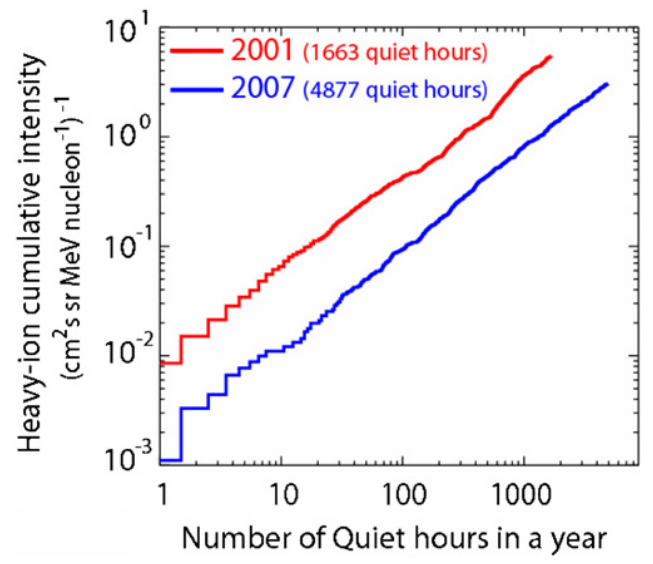

Figure 4. STEP heavy-ion cumulative intensity during the quiet hours of 2001 and 2007. The smooth increase with increasing quiet hours indicates comparable intensities during all quiet hours.

Figure 7 shows the fraction of time when ${ }^{3} \mathrm{He}$ is present during the quiet hours of every Bartels rotation from 1998 January 1 to 2007 December 1. While some Bartels rotations have no signatures of ${ }^{3} \mathrm{He}$ (vertical green arrows), other rotations show that ${ }^{3} \mathrm{He}$ is present for more than $90 \%$ of the quiet hours. On average, ${ }^{3} \mathrm{He}$ is present $\sim 27.4 \%$ of the time during the quiet hours of 1998-2004, and 5\% of those from 2004 to 2007. This sharp declination of ${ }^{3} \mathrm{He}$ toward the end of the solar cycle 23 is also observed by M. E. Wiedenbeck et al. (2005, private communication) at higher energies.

\subsection{Spectral Properties of Heavy Ions During the Quiet Times}

In order to study the spectral properties of suprathermal heavy ions during quiet times over the solar cycle, we examined the integrated spectra for $\mathrm{CNO}, \mathrm{Ne}-\mathrm{S}$, and Fe from 1995 through 2007 using STEP at energies 0.04-0.32 MeV nucleon ${ }^{-1}$ and from 1998 through 2007 using ULEIS at energies between 0.11 and $2.56 \mathrm{MeV}$ nucleon ${ }^{-1}$ during the selected quiet times. We remark that the intensities at similar energies for each species from the two instruments, on average, agreed within a factor of 5 (e.g., Fe in Figure 8(c)), with the largest difference occurring for the Ne-S spectra during 1999 (a factor of 8, not shown). To understand such differences, we re-identified the quiet times from both instruments using the same energy range between 0.1 and $0.32 \mathrm{MeV}$ nucleon $^{-1}$. For all species, the intensities during these quiet times agreed within a factor of $\sim 1.6$, indicating that the above-mentioned differences are due to the difference in the energy ranges used in our survey, and not due to instrumental effects.

We fitted STEP spectra by a power law of the form $j(E)=$ $j_{0} E^{-\gamma}$, where $j_{0}$ is the normalization constant, $E$ is the energyper-nucleon, and $\gamma$ is the spectral index. In all the cases, we fit the spectrum of each species independently by using a gridsearch technique (Bevington \& Robinson 1992) that minimized the chi-squared $\left(\chi^{2}\right)$ for each fitted parameter and yielded best parameter fits. In general, all the spectral fits were excellent visually and statistically with a reduced chi-squared ranging between 0.59 and 1.12. ULEIS energy spectra of the heavier ions (e.g., $\mathrm{Fe}$ ) tend to roll over at higher energies faster than those of

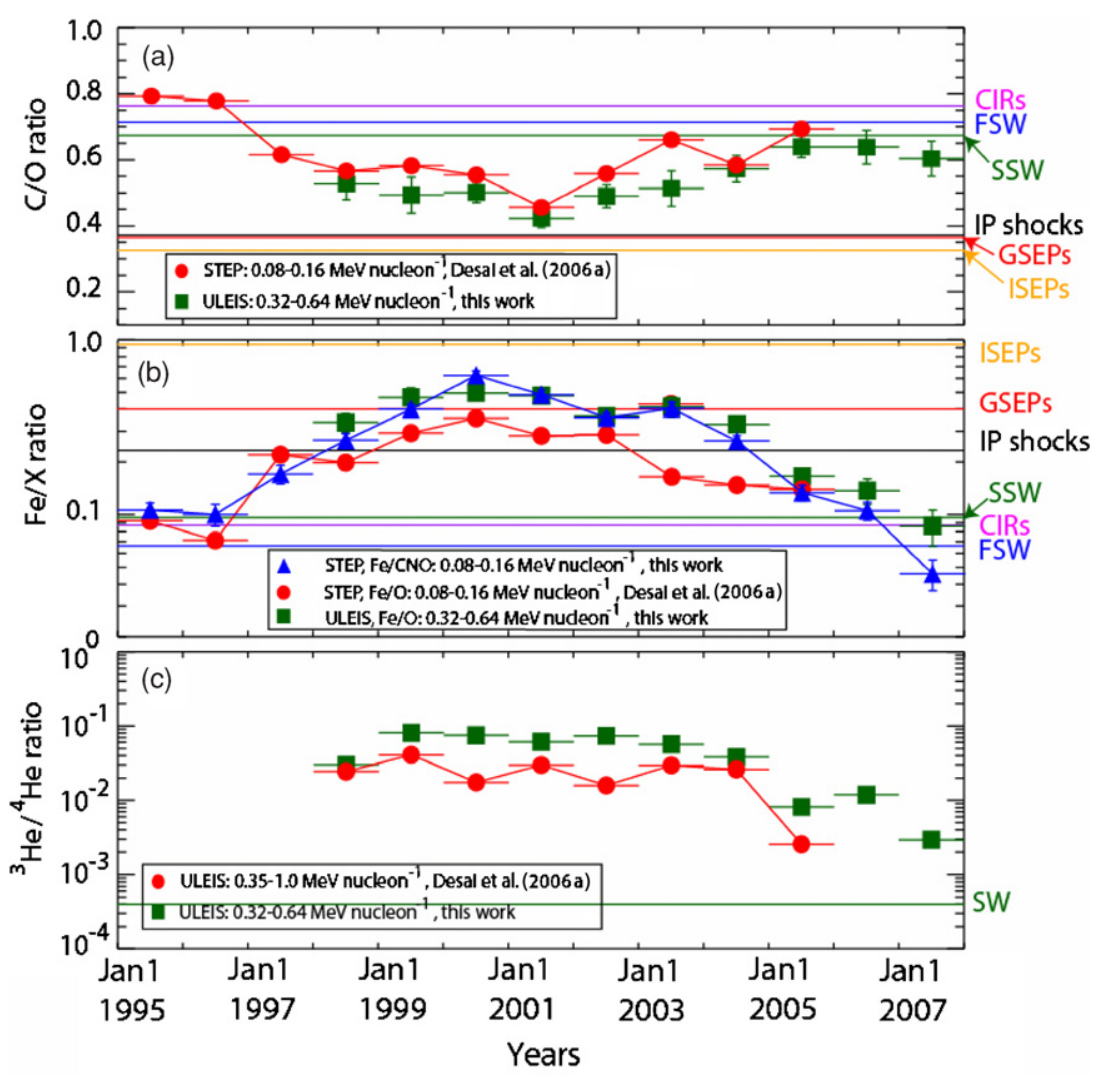

Figure 5. (a) $\mathrm{C} / \mathrm{O},(b) \mathrm{Fe} / \mathrm{O}$ and $\mathrm{Fe} / \mathrm{CNO}$, and $(c){ }^{3} \mathrm{He} /{ }^{4} \mathrm{He}$ ratios as measured by STEP and ULEIS at different energy ranges between 1995 and 2007. On all panels: the red plots are those determined by Desai et al. (2006) The horizontal lines represent averaged abundances measured in different heliosphere particle populations: CIRs abundance at $\sim 0.15 \mathrm{MeV}$ nucleon ${ }^{-1}$ (CIRs; Mason et al. 2008); ${ }^{3} \mathrm{He}$-rich or impulsive SEP events at $\sim 0.375 \mathrm{MeV}^{\text {nucleon }}{ }^{-1}$ (impulsive SEPs; Mason et al. 2002); gradual SEP events at $\sim 0.38 \mathrm{MeV}$ nucleon ${ }^{-1}$ (gradual SEPs; Desai et al. 2006b); CME-driven IP shocks at $\sim 0.75 \mathrm{MeV}^{2}$ nucleon ${ }^{-1}$ (IP shocks; Desai et al. 2003); fast SW (FSW; Gloeckler \& Geiss 2007), and slow SW (SSW; von Steiger et al. 2000). The horizontal line in panel (c) shows the average SW value (Gloeckler \& Geiss 1998). 
Table 1

Quiet-time Elemental Abundances from ULEIS at $0.32-0.64 \mathrm{MeV}$ nucleon ${ }^{-}$

\begin{tabular}{|c|c|c|c|c|c|c|c|c|c|c|}
\hline \multirow[t]{2}{*}{ Year } & \multirow[t]{2}{*}{${ }^{3} \mathrm{He} /{ }^{4} \mathrm{He}(\%)$} & \multicolumn{9}{|c|}{ Heavy Ion Abundances During Quiet Times at $0.32-0.64 \mathrm{MeV}$ nucleon $^{-1}$} \\
\hline & & $\mathrm{C}$ & $\mathrm{N}$ & $\mathrm{O}^{\mathrm{a}}$ & $\mathrm{Ne}$ & $\mathrm{Mg}$ & $\mathrm{Si}$ & S & $\mathrm{Ca}$ & $\mathrm{Fe}$ \\
\hline 1998 & $2.96 \pm 0.13$ & $0.511 \pm 0.052$ & $0.164 \pm 0.025$ & $\equiv 1 \pm 0.085$ & $0.177 \pm 0.029$ & $0.183 \pm 0.028$ & $0.264 \pm 0.036$ & $0.059 \pm 0.015$ & $\leqslant 0.027$ & $0.337 \pm 0.042$ \\
\hline 1999 & $8.05 \pm 0.35$ & $0.475 \pm 0.059$ & $0.166 \pm 0.030$ & $\equiv 1 \pm 0.100$ & $0.250 \pm 0.040$ & $0.260 \pm 0.040$ & $0.292 \pm 0.046$ & $0.062 \pm 0.016$ & $\leqslant 0.032$ & $0.469 \pm 0.062$ \\
\hline 2000 & $7.46 \pm 0.21$ & $0.483 \pm 0.032$ & $0.143 \pm 0.014$ & $\equiv 1 \pm 0.055$ & $0.198 \pm 0.018$ & $0.240 \pm 0.020$ & $0.250 \pm 0.021$ & $0.110 \pm 0.012$ & $0.035 \pm 0.007$ & $0.497 \pm 0.034$ \\
\hline 2001 & $6.08 \pm 0.24$ & $0.398 \pm 0.030$ & $0.128 \pm 0.014$ & $\equiv 1 \pm 0.058$ & $0.199 \pm 0.019$ & $0.262 \pm 0.022$ & $0.222 \pm 0.021$ & $0.071 \pm 0.010$ & $0.030 \pm 0.007$ & $0.480 \pm 0.035$ \\
\hline 2002 & $7.34 \pm 0.31$ & $0.471 \pm 0.037$ & $0.155 \pm 0.018$ & $\equiv 1 \pm 0.066$ & $0.171 \pm 0.02$ & $0.225 \pm 0.023$ & $0.240 \pm 0.025$ & $0.082 \pm 0.012$ & $0.036 \pm 0.009$ & $0.367 \pm 0.034$ \\
\hline 2003 & $5.64 \pm 0.35$ & $0.496 \pm 0.058$ & $0.149 \pm 0.027$ & $\equiv 1 \pm 0.097$ & $0.154 \pm 0.028$ & $0.186 \pm 0.032$ & $0.168 \pm 0.031$ & $0.089 \pm 0.021$ & $\leqslant 0.024$ & $0.416 \pm 0.054$ \\
\hline 2004 & $3.80 \pm 0.19$ & $0.560 \pm 0.043$ & $0.145 \pm 0.019$ & $\equiv 1 \pm 0.066$ & $0.194 \pm 0.022$ & $0.246 \pm 0.026$ & $0.204 \pm 0.024$ & $0.060 \pm 0.012$ & $0.047 \pm 0.01$ & $0.329 \pm 0.032$ \\
\hline 2005 & $0.79 \pm 0.06$ & $0.632 \pm 0.035$ & $0.135 \pm 0.013$ & $\equiv 1 \pm 0.049$ & $0.208 \pm 0.017$ & $0.158 \pm 0.015$ & $0.138 \pm 0.014$ & $0.046 \pm 0.009$ & $\leqslant 0.014$ & $0.165 \pm 0.016$ \\
\hline 2006 & $1.16 \pm 0.11$ & $0.632 \pm 0.056$ & $0.113 \pm 0.019$ & $\equiv 1 \pm 0.079$ & $0.261 \pm 0.031$ & $0.113 \pm 0.019$ & $0.118 \pm 0.021$ & $0.043 \pm 0.010$ & $\leqslant 0.027$ & $0.137 \pm 0.023$ \\
\hline 2007 & $0.28 \pm 0.05$ & $0.594 \pm 0.057$ & $0.128 \pm 0.022$ & $\equiv 1 \pm 0.083$ & $0.250 \pm 0.032$ & $0.130 \pm 0.022$ & $0.097 \pm 0.020$ & $\leqslant 0.022$ & $\leqslant 0.012$ & $0.086 \pm 0.020$ \\
\hline
\end{tabular}

Note. ${ }^{a}$ Normalization. 

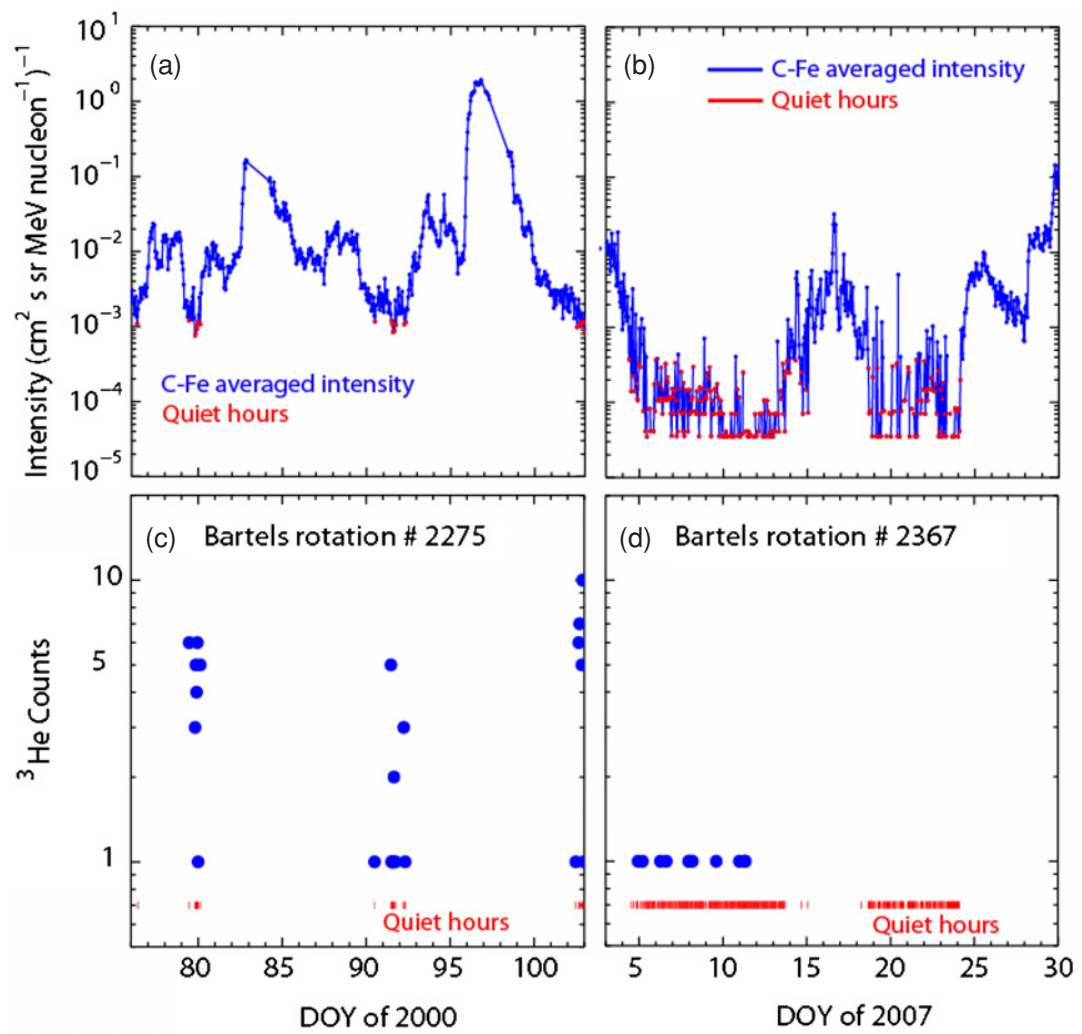

Figure 6. $(a)$ and $(b)$ Heavy-ion intensity for Bartels rotations 2275 (days 76-103 of 2000) and 2367 (days 3-30 of 2007), respectively. Intensities during selected quiet hours are plotted in red. $(c)$ and $(d)$ Hourly ${ }^{3} \mathrm{He}$ counts during the quiet hours of the selected Bartels rotations. The red dots correspond to the quiet hours during each of the 27 day periods.

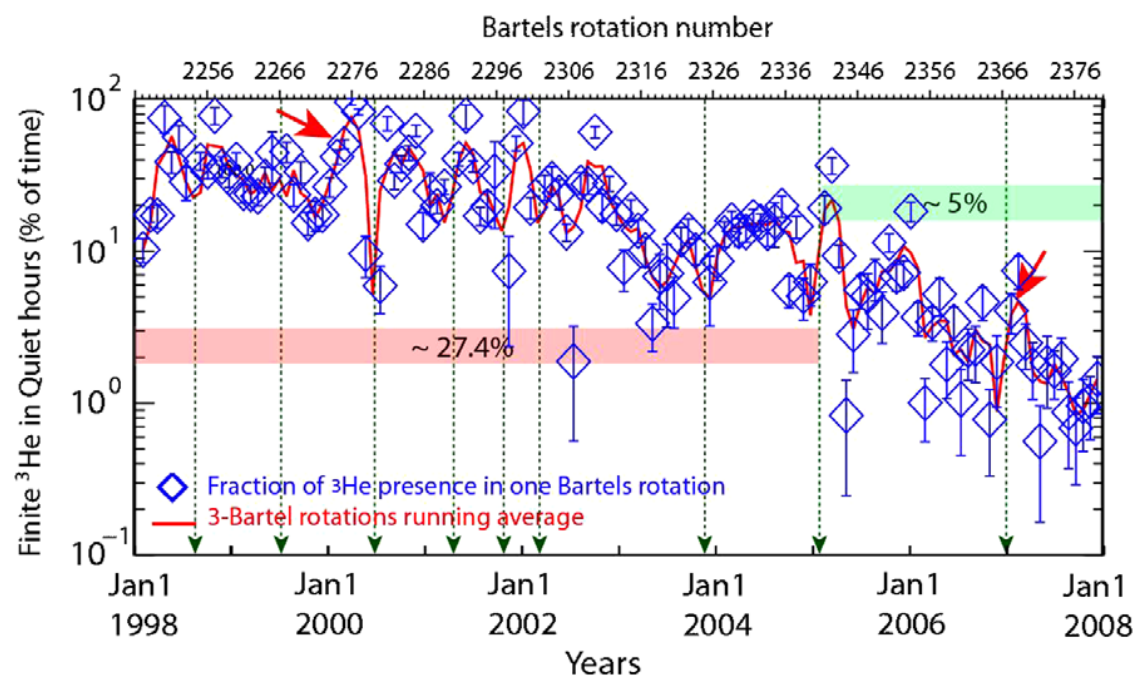

Figure 7. (a) Fraction of time with finite ${ }^{3} \mathrm{He}$ during the quiet times from 1998 to 2007. Points are shown for individual Bartels rotations; red line shows the running average over three rotations. Vertical green arrows indicate rotations with no signatures of ${ }^{3} \mathrm{He}$ and red arrows point to the Bartels rotations shown in Figure 6.

lighter ones (e.g., CNO). To account for such roll-overs, we fitted the ULEIS spectra by a power law modulated by an exponential of the form $j(E)=j_{0} E^{-\gamma} \exp \left(-\frac{E}{E_{0}}\right)$ where $\gamma$ is the spectral index and $E_{0}$ is the e-folding energy (e.g., Jones \& Ellison 1991; Ruffolo \& Channok 2003). This characteristic form (hereafter the JE expression) has been successful in representing the energy spectra of ions during several impulsive and gradual SEP events (Ellison \& Ramaty 1985; Tylka et al. 2000; Klecker et al. 2003; Desai et al. 2004). STEP and ULEIS spectral indices and e-folding energies for different species for each year are respectively shown in Table 2 .

\subsubsection{Solar Cycle Dependence}

Figure 8 shows the energy spectra of $\mathrm{CNO}, \mathrm{Ne}-\mathrm{S}$, and $\mathrm{Fe}$ of $\mathrm{Fe} / \mathrm{CNO}$ for 3 years corresponding to the minima and maxima of solar activity: (a) 1996, (b) 2001, and (c) 2007. Figures 8(b) and 8(c) show that ULEIS quiet-time energy spectra roll over at higher energies, and that the intensities at lower energies are consistent with those from STEP at similar energies.

Figures 8(d)-(f) show the energy dependence of $\mathrm{Fe} / \mathrm{CNO}$ during the quiet times of the three different years, along with the averaged $\mathrm{Fe} / \mathrm{CNO}$ ratios measured in different populations (as in Figure 5). As shown, the $\mathrm{Fe} / \mathrm{CNO}$ ratio exhibits a clear 

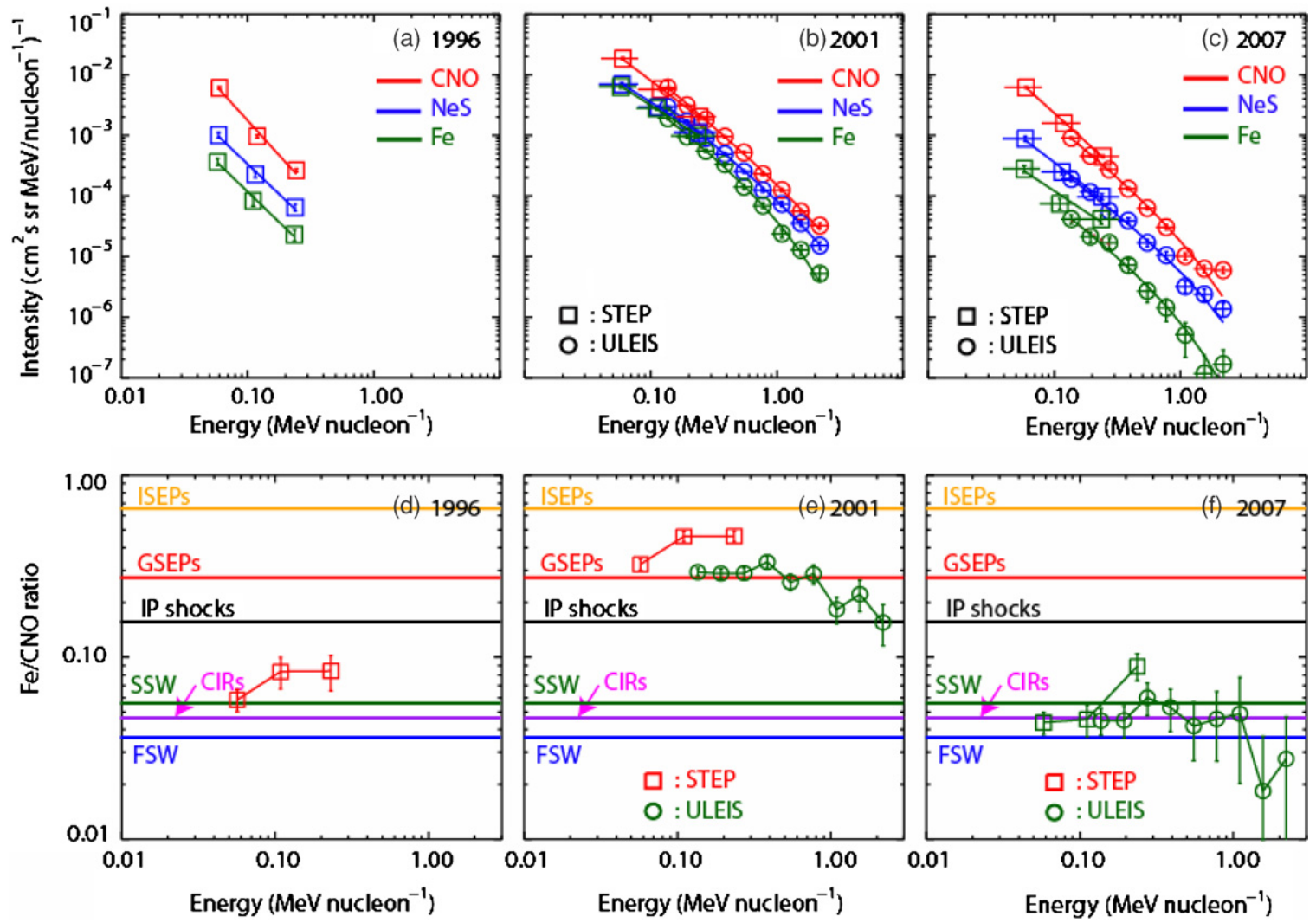

Figure 8. ULEIS (squares) and STEP (circles)-integrated energy spectra of heavy ions during the quiet times of (a) 1996 (STEP), (b) 2001, and (c) 2007. The solid lines represent the best fits. All STEP data points are fitted by a power law and all ULEIS data points are fitted with the Jones and Ellison expression where the spectral index and the e-folding energy are determined (see the text for details). Panels $(d)-(f)$ : energy dependence of Fe/CNO during the quiet times of 1996, 2001, and 2007. Horizontal lines: averaged abundances of Fe/CNO in different heliospheric populations (as in Figure 5).

Table 2

Spectral Indices and e-Folding Energies from STEP and ULEIS Fitted Energy Spectra

\begin{tabular}{|c|c|c|c|c|c|c|c|c|c|}
\hline \multirow[t]{3}{*}{ Year } & \multicolumn{3}{|c|}{$\mathrm{CNO}$} & \multicolumn{3}{|c|}{$\mathrm{NeS}$} & \multicolumn{3}{|c|}{$\mathrm{Fe}$} \\
\hline & \multirow{2}{*}{$\begin{array}{l}\text { STEP } \\
\gamma^{\mathrm{a}}\end{array}$} & \multicolumn{2}{|c|}{ ULEIS } & \multirow{2}{*}{$\begin{array}{c}\text { STEP } \\
\gamma^{\mathrm{a}}\end{array}$} & \multicolumn{2}{|c|}{ ULEIS } & \multirow{2}{*}{$\begin{array}{c}\text { STEP } \\
\gamma^{\mathrm{a}}\end{array}$} & \multicolumn{2}{|c|}{ ULEIS } \\
\hline & & $E_{0}^{\mathrm{b}, \mathrm{c}}$ & $\gamma^{\mathrm{b}}$ & & $E_{0}^{\mathrm{b}, \mathrm{c}}$ & $\gamma^{\mathrm{b}}$ & & $E_{0}^{\mathrm{b}, \mathrm{c}}$ & $\gamma^{\mathrm{b}}$ \\
\hline 1996 & $2.29 \pm 0.05$ & $\mathrm{~N} / \mathrm{A}$ & $\mathrm{N} / \mathrm{A}$ & $1.98 \pm 0.12$ & $\mathrm{~N} / \mathrm{A}$ & $\mathrm{N} / \mathrm{A}$ & $2.01 \pm 0.18$ & $\mathrm{~N} / \mathrm{A}$ & $\mathrm{N} / \mathrm{A}$ \\
\hline 1997 & $2.01 \pm 0.05$ & $\mathrm{~N} / \mathrm{A}$ & $\mathrm{N} / \mathrm{A}$ & $1.60 \pm 0.1$ & $\mathrm{~N} / \mathrm{A}$ & $\mathrm{N} / \mathrm{A}$ & $1.91 \pm 0.10$ & $\mathrm{~N} / \mathrm{A}$ & $\mathrm{N} / \mathrm{A}$ \\
\hline 1998 & $1.56 \pm 0.05$ & $\ldots$ & $1.85 \pm 0.03$ & $1.48 \pm 0.08$ & $\ldots$ & $1.90 \pm 0.04$ & $1.79 \pm 0.22$ & $1.75 \pm 0.32$ & $1.77 \pm 0.06$ \\
\hline 2001 & $1.57 \pm 0.04$ & $\ldots$ & $1.72 \pm 0.02$ & $1.32 \pm 0.06$ & $\ldots$ & $1.67 \pm 0.03$ & $1.33 \pm 0.06$ & $1.08 \pm 0.06$ & $1.55 \pm 0.03$ \\
\hline 2002 & $1.46 \pm 0.06$ & $\ldots$ & $1.73 \pm 0.02$ & $1.28 \pm 0.08$ & $\ldots$ & $1.75 \pm 0.03$ & $1.64 \pm 0.07$ & $1.06 \pm 0.08$ & $1.59 \pm 0.04$ \\
\hline 2003 & $1.75 \pm 0.07$ & $\ldots$ & $1.60 \pm 0.03$ & $1.75 \pm 0.1$ & $\ldots$ & $1.85 \pm 0.05$ & $1.67 \pm 0.09$ & $1.53 \pm 0.22$ & $1.45 \pm 0.07$ \\
\hline 2004 & $1.80 \pm 0.03$ & $\ldots$ & $1.61 \pm 0.02$ & $1.71 \pm 0.06$ & $\ldots$ & $1.49 \pm 0.04$ & $1.65 \pm 0.07$ & $1.34 \pm 0.13$ & $1.47 \pm 0.05$ \\
\hline 2005 & $1.38 \pm 0.03$ & $1.58 \pm 0.05$ & $1.32 \pm 0.02$ & $1.46 \pm 0.06$ & $1.74 \pm 0.11$ & $1.36 \pm 0.03$ & $1.58 \pm 0.08$ & $1.92 \pm 0.27$ & $1.65 \pm 0.05$ \\
\hline 2006 & $1.79 \pm 0.04$ & $1.57 \pm 0.1$ & $1.85 \pm 0.02$ & $1.84 \pm 0.1$ & $\ldots$ & $1.87 \pm 0.04$ & $1.35 \pm 0.12$ & & $1.69 \pm 0.09$ \\
\hline
\end{tabular}

Notes.

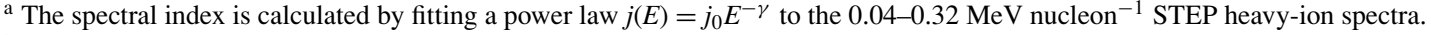

$\mathrm{b}$ e-folding energy $E_{0}$ is obtained by fitting the $0.11-2.56 \mathrm{MeV}$ nucleon ${ }^{-1}$ ULEIS heavy-ion spectra with the $\operatorname{spectral~form~} j(E)=j_{0} E^{-\gamma} \exp \left(-E / E_{0}\right)$.

${ }^{c}$ Values are provided as of 1998 (ULEIS data). Ellipsis values refer to roll-overs beyond the data energy range.

dependence on solar activity, with the $\mathrm{Fe} / \mathrm{CNO}$ ratio varying by almost an order of magnitude between 2001 and 2007. Comparing with the spectra in Figures 8(a)-(c) we note that the compositional differences between solar minimum and solar maximum are largely due to the significantly larger intensities of Fe in the IP medium. In particular, we note that: (1) during solar minimum the $\mathrm{Fe} / \mathrm{CNO}$ ratio is similar (and slightly lower in 2007) to that observed in the SW and CIRs, while during solar maximum period it increases to the values observed in gradual SEPs and IP shocks, as also shown in Figure 5(b). (2) Interestingly, Figures $8(a)-(c)$ show that the CNO intensity level varies by less than a factor of 2 during the 3 years of 

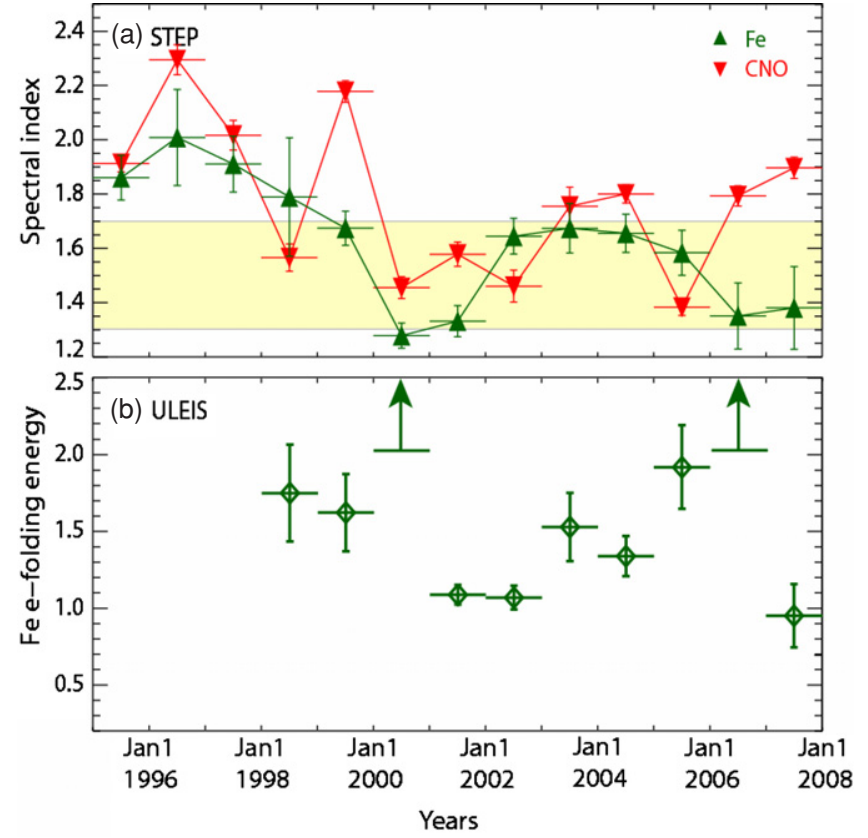

Figure 9. (a) Variations of the quiet-time spectral indices of $\mathrm{CNO}$ and $\mathrm{Fe}$ as measured by STEP for years $1995-2007$ at $0.04-0.32 \mathrm{MeV}$ nucleon ${ }^{-1}$. The highlighted regions mark index values between 1.3 and 1.7. (b) e-folding energy variations of the quiet-time Fe spectra from 1998 to 2007 obtained by fitting the JE spectral expression (the arrows indicate that the roll-over occurred beyond the fitted data range).

different solar activity, while Fe intensities increase by almost an order of magnitude during 2001 when compared with the values observed during 1996 and 2007. In addition, Figure 8(e) shows that ULEIS Fe/CNO ratio remains constant at energies below $0.5 \mathrm{MeV}$ nucleon $^{-1}$ and tends to decrease above $\sim 1$ $\mathrm{MeV}$ nucleon ${ }^{-1}$. Such energy dependences (also clear in 2000 , not shown) are only observed at the higher energies because they are due to the spectral roll-overs.

To investigate the solar cycle variations of the quiet-time energy spectra, we plot in Figure 9(a) CNO and Fe STEP spectral indices from 1995 to 2007, and (b) ULEIS e-folding energies of Fe during 1998 through 2007 from Table 2. In 10 of the 13 years, the CNO spectra is softer than that of Fe, with 3 years (1998, 2002 , and 2005) showing a harder CNO spectrum. Additionally, the Fe spectra during the first solar minimum (1995-1997) are softer than those observed during the second solar minimum (2005-2007). The hardest Fe spectrum occur during the solar maximum years (2000 and 2001) which is consistent with the higher Fe intensity levels seen in Figure 8(b) and the higher Fe/ CNO ratios seen in Figures 5(b) and 8(e). Overall, the spectral indices of $\mathrm{CNO}$ and Fe fluctuate largely for different years with no clear solar cycle dependence.

Figure 9(b) shows that the e-folding energies of the extended energy spectra measured by ULEIS also fluctuate over the solar cycle with no clear trend, and that the Fe spectrum during 2000 and 2006 rolled over above $\sim 2.5 \mathrm{MeV}$ nucleon $^{-1}$. We also repeated our analysis by increasing/decreasing the fraction of selected quiet-hours by $5 \%$ and found that the results remained unchanged when compared with those reported here. However, when we increased the threshold by $10 \%$ or more (e.g., for 2000 using $30 \%$ of the quietest hours instead of $20 \%$ ), we observed clearer solar cycle dependence with higher e-folding energies occurring during solar maximum years and lower e-folding energies occurring during years of lower solar activity levels. This solar cycle dependence of the roll-over is probably due to the
$\mathrm{M} / \mathrm{Q}$ dependence of the acceleration processes occurring in large SEP and CME-shock-accelerated events (see Desai et al. 2004). Thus, it appears that by including a larger fraction of hours during solar maximum, the "quiet hours" can be "contaminated" by heavy ions that were most likely accelerated in CME-shock-related events. This provides us with additional confidence that our selected quiet hours yield a reasonable measure of the suprathermal population properties during all solar activity levels.

\subsubsection{Energy Spectra}

Figure 10 investigates the relationship between the 0.04 $0.32 \mathrm{MeV}$ nucleon $^{-1}$ spectral indices of $\mathrm{CNO}, \mathrm{Ne}-\mathrm{S}$, and $\mathrm{Fe}$ species measured by STEP during the quiet times and listed in Table 2. As shown, the correlation between $\mathrm{Ne}-\mathrm{S}$ spectral indices and that of CNO (correlation coefficient $(r)=0.78$ ) is larger than that of Fe with $\mathrm{CNO}(r=0.53)$. Moreover, Figure 10 shows that at energies between 0.04 and $0.32 \mathrm{MeV}$ nucleon $^{-1}$, heavier elements (i.e., $\mathrm{Fe}$ ) have, in general, harder spectra than those with lighter mass (i.e., CNO). We note that Fe spectral indices vary between 1.27 and 2.01, while those of CNO fluctuate between 1.38 and 2.29. The yellow-highlighted regions in the figure mark index values between 1.3 and 1.7. Note that of the 13 years, 8 of the Fe spectral indices, 7 of the Ne-S, and 5 of the CNO indices lie within this range, and can therefore be considered to be close to $\sim 1.5$. We remark that when we tried different selection criteria for the quiet times, we obtained spectral indices that were within the statistical uncertainties of those shown in Table 2.

\section{DISCUSSION}

We have analyzed the quiet time composition and energy spectra of heavy ions in the suprathermal energy range between 0.04 and $0.32 \mathrm{MeV}$ nucleon $^{-1}$ for years 1995-2007 and between 0.11 and $2.56 \mathrm{MeV}^{\text {nucleon }}{ }^{-1}$ from 1998 to 2007 using STEP and ULEIS, respectively. Our results show that:

1. Heavy-ion elemental abundances ( $\mathrm{C}$ through $\mathrm{Fe}$ ) at energies $0.32-0.64 \mathrm{MeV}$ nucleon $^{-1}$ during the quiet times of the solar cycle 23 are similar to the average abundances observed in SEP and IP shock events during solar maximum, and SW and CIR abundances during solar minimum (Figures 5(a) and (b), and Figure 8(d)-(f)).

2. The ${ }^{3} \mathrm{He} /{ }^{4} \mathrm{He}$ ratio varies between $3 \%$ and $8 \%$ during the quiet times of 1998-2004 with an averaged ${ }^{3} \mathrm{He}$ abundance of $\sim 27.4 \%$ of the quiet time. This ratio drops significantly by more than an order of magnitude $(0.3 \%-1.2 \%)$ during the quiet times of 2005-2007 with a significant drop of ${ }^{3} \mathrm{He}$ abundance to $\sim 5 \%$ (Figures 5(c) and 7).

3. All heavy-ion species ( $\mathrm{C}$ through $\mathrm{Fe}$ ) have suprathermal tails between 0.04 and $0.32 \mathrm{MeV}$ nucleon ${ }^{-1}$ with spectral indices ranging from $\sim 1.27$ to $\sim 2.29$. These tails sometimes extend above $\sim 2 \mathrm{MeV}$ nucleon ${ }^{-1}$ (Figures 8 and $9(\mathrm{~b})$ ) with heavy-ion $(\mathrm{Fe})$ spectra rolling over at lower energies than lighter ones (CNO).

4. The suprathermal spectral indices of different species at $0.04-0.32 \mathrm{MeV}$ nucleon $^{-1}$ are correlated in a way that heavier elements (i.e., $\mathrm{Fe}$ ) have harder spectra than the lighter ones (i.e., CNO). These indices fluctuate without exhibiting clear solar cycle dependence and for $\sim 50 \%$ of the time, the spectral indices of heavy ions deviate significantly from the 1.5 value (Figures 9 and 10). 

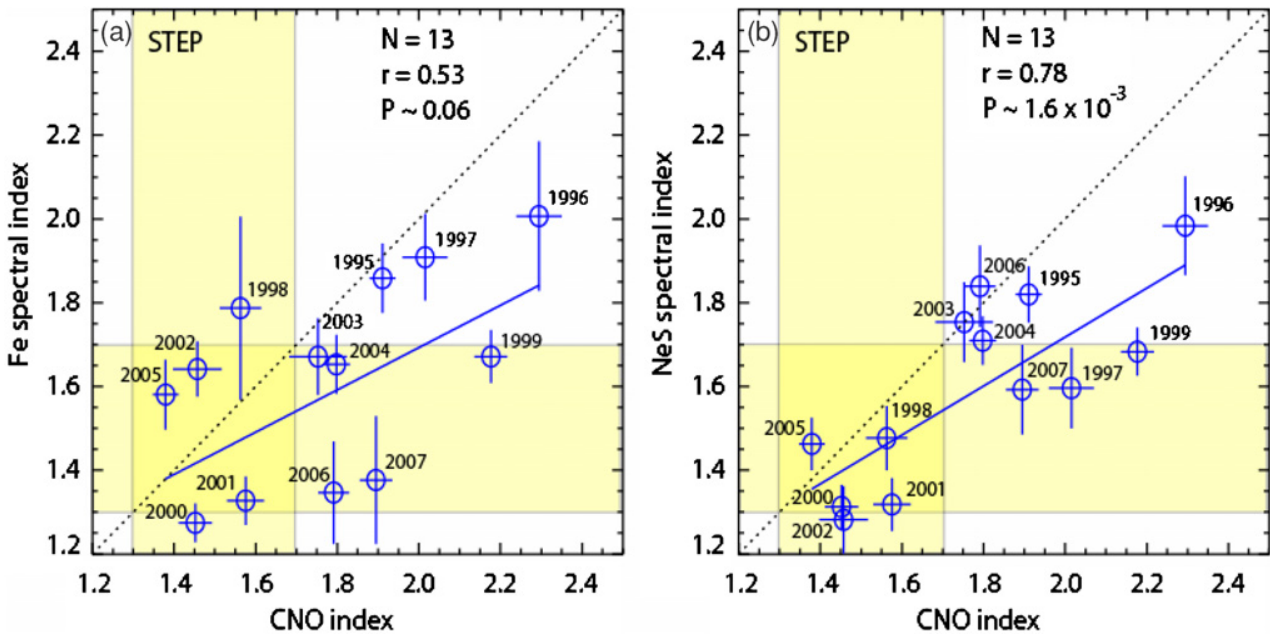

Figure 10. Power-law spectral indices of Fe $(a)$ and Ne-S $(b)$ vs. that of CNO as measured by Wind/STEP at $0.04-0.32 \mathrm{MeV}$ nucleon ${ }^{-1}$ for years $1995-2007$. The highlighted regions indicate index values between 1.3 and 1.7 .

There are at least two hypotheses regarding the origin of these IP suprathermal ions, namely, (1) the ions are the low-energy component of particles that are accelerated during prior and ongoing solar and IP activity (Desai et al. 2006a; Mewaldt et al. 2006), and/or (2) the suprathermal ions are accelerated via some kind of stochastic acceleration mechanisms operating in the IP medium (Fisk \& Gloeckler 2006, 2007a; Gloeckler et al. 2008). Both these notions have specific expectations for the observations. In the former scenario, the dominant component of the suprathermal material is expected to vary according to the phase of the solar cycle. In other words, ions accelerated during solar energetic particles or SEPs should dominate the composition during solar maximum while ions from CIRs or from the suprathermal tail of the SW should dominate during solar minimum periods. In the latter scenario, there is no a priori reason for the suprathermal heavy-ion composition to vary with solar activity and the low-energy portion (up to $\sim$ $0.1 \mathrm{MeV}$ nucleon $^{-1}$ ) of the differential energy spectrum should exhibit a power law with a fixed value of 1.5 for the spectral index.

During all the quiet hours in our survey, the $\mathrm{Fe} / \mathrm{O}$ and $\mathrm{C} / \mathrm{O}$ ratios exhibit a clear dependence on the solar cycle with SEPlike abundances during solar maximum and SW or CIR-like composition during solar minimum (Figure 5). The depletion of Fe (see Figures 8(b) and (c)) during the solar minimum quiet hours is likely due to the sharp decrease in flare activity (see Figure 1(c)), with the ion abundances probably reflecting the SW (von Steiger et al. 2000; Gloeckler \& Geiss 2007) or CIR composition (Mason et al. 2008). This picture is also consistent with the frequent presence and higher abundance of ${ }^{3} \mathrm{He}$ in IP space during both the quiet (Figure 7) and nonquiet times (Wiedenbeck et al. 2003, 2005) of solar maximum from 1998 to 2003. These results are in agreement with recent work by Desai et al. (2006a) who showed that the elemental composition of $\sim 0.08-0.16 \mathrm{MeV}$ nucleon ${ }^{-1}$ suprathermal population during quiet times (defined by setting a threshold on Fe intensity) is also strongly correlated with solar activity. Figure 8(e) also shows that the $\mathrm{Fe} / \mathrm{CNO}$ ratio decreases with increasing energies (also during 2000, not shown). Such behavior is often observed in large SEP events and CME-driven IP shock events (see Desai et al. 2004, 2006a) and is generally attributed to rigidity or M/Qdependent processes occurring during acceleration/escape from the CME shock acceleration region (e.g., Cohen et al. 2005; Mason et al. 2006).
On this basis, we conclude that during the quiet times of solar maximum from 1998 to 2004, the suprathermal heavyion population is dominated by an admixture of ions that are accelerated in ${ }^{3} \mathrm{He}$-rich and Fe-rich impulsive SEPs and by CME-driven shocks in the inner heliosphere. Since the occurrence rates of flares and CMEs vary significantly from one Bartels rotation to the next, the amount, composition, and spectral properties of the suprathermal material in IP space is also highly variable and dynamic. In contrast, during solar minimum periods, the occurrence rates of flares and CMEs decline significantly and so the relative contributions of SEP material to the suprathermal ion population is significantly lower. Thus, the suprathermal heavy-ion composition resembles that measured in the two dominant sources- the heated SW and previously accelerated CIR material.

Fisk \& Gloekler (2006, 2007a, 2007b; also Gloeckler et al. 2008) recently reported that suprathermal tails exhibit unique spectral index of 1.5 when expressed as differential intensity as a function of energy. They suggested that these ubiquitous spectral tails could be produced by stochastic acceleration due to compressional turbulence in the SW and that heavier ions (e.g., $\mathrm{He}$ ) roll over at energies lower than that of lighter ions (e.g., protons). Our results show that although heavy ions exhibit suprathermal tails during quiet times, the spectral indices for 0.04-0.32 MeV nucleon ${ }^{-1}$ heavy ions vary over a wide range between 1.27 and 2.29 for $\mathrm{Fe}$ and CNO. In other words, the theoretical prediction of the ubiquitous 1.5 suprathermal spectral index reported by Fisk \& Gloeckler does not appear to be a special case and is therefore somewhat at odds with our observations.

It is worthwhile noting that the $\mathrm{Fe}$ and $\mathrm{CNO}$ spectral indices are closer to the predicted 1.5 value during solar maximum years. This is probably a coincidence since our composition results clearly indicate that the suprathermal population greater than $0.04 \mathrm{MeV}$ nucleon $^{-1}$ is essentially dominated by SEP-like material which is most likely accelerated near the sun and inside $1 \mathrm{AU}$ during flares and CME-driven shock events, rather than from stochastic acceleration mechanisms occurring in the IP medium. On the other hand, during solar minimum years of 1995-1997 when the flare and CME activity is low and therefore their contributions to the suprathermal population are sufficiently reduced, the spectral indices of $\mathrm{CNO}$ and $\mathrm{Fe}$ lie between a wide range from $\sim 1.6$ to 2.3 . In other words, given the SW-like and CIR-like composition signatures observed during periods of 
low solar activity, one would expect the dominant contributions to the suprathermal population to originate from IP acceleration processes like the one proposed by Fisk \& Gloeckler, and yet it is during these periods that the Fe and CNO spectral indices deviate most significantly from the predicted 1.5 value.

The differences between our results and those of Fisk \& Gloeckler could be due a combination of the following four reasons. First, they used the SWICS instruments on board Ulysses and $A C E$ that measure ions with energies up to $\sim 60$ and $\sim 100 \mathrm{keV} \mathrm{e}^{-1}$ respectively. Although our derived spectral indices cover a significantly higher energy range between 40 and $320 \mathrm{keV}^{\text {nucleon }}{ }^{-1}$, the lower energy portion of our spectra overlaps with the upper energy range of the SWICS instruments. Thus, since we use higher energies to derive our indices, the difference in spectral indices might be due to steepening above $\sim 200 \mathrm{keV}$ nucleon ${ }^{-1}$. However, we believe that this difference is unlikely to affect our results significantly and account for the observed differences because in all cases reported here we found that the heavy-ion spectra between $\sim 0.04$ and $0.32 \mathrm{MeV}$ nucleon $^{-1}$ are well fit by a single power law and that the steepening or roll-over of the spectra occurs only when we included the ULEIS data above $0.5 \mathrm{MeV}$ nucleon $^{-1}$ (see Table 2 and Figure 9(b)). Second, we note that the 1.5 spectral index is a prediction for steady-state acceleration and the presence of steeper spectra might be an indication that equilibrium has not been reached (see also Mason et al. 2008). We remark that although this explanation could account for the apparent discrepancy between the theoretical prediction and our observations, it cannot account for the differences in the observational results from the two studies, which show highly variable spectral indices in our case compared with a unique 1.5 in the study by Fisk \& Gloeckler. Third, we specifically looked at the spectral index in the energy range between 0.04 and $0.32 \mathrm{MeV}$ nucleon $^{-1}$ obtained from a power-law fit. Fisk \& Gloeckler on the other hand, fitted an extended energy spectrum between $\sim 0.03$ and $\sim 2 \mathrm{MeV}$ nucleon ${ }^{-1}$ with a fixed spectral index of 1.5 for the lower energy portion and two free parameters to describe the roll-over at higher energies of the spectrum. In particular, they used a power law modulated with an exponential and a rigidity dependent roll-over energy (see Gloeckler et al. 2008).

Finally, we note that our selection criteria of the quiet times is somewhat different from that used in the Gloeckler \& Fisk study; their quiet times are periods where the SW speed is less than $320 \mathrm{~km} \mathrm{~s}^{-1}$ (Gloeckler et al. 2008). We believe that most of the differences between the observational results could be attributed to the selection criteria of the quiet times in the two studies. However, at present, we cannot quantify the consequences of using different selection criteria. A more thorough investigation of this issue is beyond the scope of this study. To fully reconcile the results from the different suprathermal ion instruments, we eventually need to include measurements from other instruments such as Wind/STICS and ACE/SWICS.

In summary, our results show that the quiet-time suprathermal heavy-ion population is highly variable and includes ions from multiple sources such as impulsive and gradual SEPs, CIRs and the SW that consistently modify their characteristics and contributions with the solar cycle activity. In particular, the suprathermal population is dominated by CIR-like or SW-like material during solar minimum conditions of 1995-1997 and 2005-2008, while it is dominated by SEP-like material during solar maximum conditions of 1998-2004. The quiet-time suprathermal heavy-ion energy spectra show significant deviations from the 1.5 value predicted by Fisk \& Gloeckler indicating that the suprathermal tails greater than $0.04 \mathrm{MeV}$ nucleon $^{-1}$ may not be dominated by the IP stochastic acceleration mechanisms that tend to produce unique spectral indices of 1.5.

We are grateful to the members of the Space Physics Group at the University of Maryland and the Johns Hopkins Applied Physics Laboratory (JHU/APL) for the construction of the ULEIS instrument. We thank the many individuals at the University of Maryland, and the EPACT instrumentation team at the Goddard Space Flight Center for the construction of the Wind/STEP instrument. Work at SwRI is partially supported by NSF grants ATM-0550960 and ATM-0551127 and NASA grants: NNG05GM88G, NNG05GQ94G, NNX07AC12G, NNX07AG85G, NNX07AP69G, NNX07AC15G, and NNX08AK87G. Part of this work has been done at Florida Institute of Technology and was supported by NSF SHINE grant 0203252 and NASA grant NAG5-11921.

\section{REFERENCES}

Acuña, M. H., Ogilvie, K. W., Baker, D. N., Curtis, S. A., Fairfield, D. H., \& Mish, W. H. 1995, Space Sci. Rev., 71, 5

Bell, A. R. 1978, MNRAS, 182, 147

Bevington, P. R., \& Robinson, D. K. 1992, in Data Reduction and Error Analysis for the Physical Sciences (2nd ed.; New York: McGraw-Hill)

Blandford, R. D., \& Ostriker, J. P. 1978, ApJ, 221, L29

Chotoo, K., et al. 2000, J. Geophys. Res., 105, 23107

Cohen, C. M. S., et al. 2000, in AIP Conf. Proc. 528, Acceleration and Transport of Energetic Particles Observed in the Heliosphere, ed. R. A. Mewaldt et al. (Melville, NY: AIP), 55

Cohen, C. M. S., et al. 2005, J. Geophys. Res., 110, A09S16

Desai, M. I., Mason, G. M., Dwyer, J. R., Mazur, J. E., Smith, C. W., \& Skoug, R. M. 2001, ApJ, 553, L89

Desai, M. I., Mason, G. M., Mazur, J. E., \& Dwyer, J. R. 2006a, ApJ, 645, L81 Desai, M. I., Mason, G. M., Müller-Mellin, R., Korth, A., Mall, U., Dwyer, J. R., \& von Rosenvinge, T. T. 2008, J. Geophys. Res., 113, A08103

Desai, M. I., et al. 2003, ApJ, 588, 1149

Desai, M. I., et al. 2004, ApJ, 611, 1156

Desai, M. I., et al. 2006b, ApJ, 649, 470

Dwyer, J. R., Mason, G. M., Desai, M. I., Mazur, J. E., \& von Rosenvinge, T. T. 2000, Geophys. Res. Lett., 27, 65

Ellison, D. C., \& Ramaty, R. 1985, ApJ, 298, 400

Fisk, L. A. 1976, J. Geophys. Res., 81, 4633

Fisk, L. A., \& Gloeckler, G. 2006, ApJL, 640, L79

Fisk, L. A., \& Gloeckler, G. 2007a, PNAS, 104, 5749

Fisk, L. A., \& Gloeckler, G. 2007b, Space Sci. Rev., 130, 153

Giacalone, J., Jokipii, J. R., \& Kóta, J. 2002, ApJ, 573, 845

Gloeckler, G. 2003, in AIP Conf. Proc. 679, Solar Wind Ten, ed. M. Velli, R. Bruno, \& F. Malara (Melville, NY: AIP), 583

Gloeckler, G., \& Geiss, J. 1998, Space Sci. Rev., 84, 275

Gloeckler, G., \& Geiss, J. 2007, Space Sci. Rev., 130, 139

Gloeckler, G., Fisk, L. A., Mason, G. M., \& Hill, M. E. 2008, in AIP Conf. Proc. 1039, Particle Acceleration and Transport in the Heliosphere and Beyond: 7th Ann. Int. Astrophysics Conf., ed. G. Li et al. (Melville, NY: AIP), 367

Gloeckler, G., Fisk, L. A., Zurbuchen, T. H., \& Schwadron, N. A. 2000, in AIP Conf. Proc. 528, Acceleration and Transport of Energetic Particles Observed in the Heliosphere: Proc. of the ACE-2000 Symp., ed. R. A. Mewaldt et al. (Melville, NY: AIP), 221

Gloeckler, G., et al. 1994, J. Geophys. Res., 99, 17637

Jones, F. C., \& Ellison, D. C. 1991, Space Sci. Rev., 58, 259

Klecker, B., et al. 2003, in Proc. 28th Int. Cosmic Ray Conf. 6/7, ed. T. Kajita et al. (Tokyo: Universal Academy Press), 3277

Kocharov, L., Laivola, J., Mason, G. M., Didkovsky, L., \& Judge, D. L. 2008, ApJS, 176, 497

Kucharek, H., et al. 2003, J. Geophys. Res., 108, LIS 15-1

le Roux, J. A., Matthaeus, W. H., \& Zank, G. P. 2001, Geophys. Res. Lett., 28, 3831

le Roux, J. A., Zank, G. P., \& Matthaeus, W. H. 2002, J. Geophys. Res., 107, SSH 9-1

Mason, G. M. 2000, in AIP Conf. Proc. 528, Acceleration and Transport of Energetic Particles Observed in the Heliosphere, ed. R. A. Mewaldt et al. (Melville, NY: AIP), 234 
Mason, G. M., Desai, M. I., Cohen, C. M. S., Mewaldt, R. A., Stone, E. C., \& Dwyer, J. R. 2006, ApJ, 647, L65

Mason, G. M., Mazur, J. E., \& Dwyer, J. R. 1999, ApJ, 525, L133

Mason, G. M., Mazur, J. E., Dwyer, J. R., Jokipii, J. R., Gold, R. E., \& Krimigis, S. M. 2004, ApJ, 606, 555

Mason, G. M., et al. 1998, Space Sci. Rev., 86, 409

Mason, G. M., et al. 2002, ApJ, 574, 1039

Mason, G. M., et al. 2008, ApJ, 678, 1458

Mazur, J. E., Mason, G. M., Dwyer, J. R., Giacalone, J., Jokipii, J. R., \& Stone, E. C. 2000, ApJ, 532, L79

Mewaldt, R. A., Cohen, C. M. S., \& Mason, G. M. 2006, in Geophysical Monograph Ser. 165, Solar Eruptions and Energetic Particles, ed. N Gopalswamy, R. A. Mewaldt, \& R. B. Torbert (Washington, DC: AGU), 115

Mewaldt, R. A., et al. 2007, Space Sci. Rev., 130, 207

Möbius, E., Hovestadt, D., Klecker, B., Scholer, M., \& Gloeckler, G. 1985 , Nature, 318, 426

Ruffolo, D., \& Channok, C. 2003, in Proc. 28th Int. Cosmic Ray Conf. 6/7, ed. T. Kajita et al. (Tokyo: Universal Academy Press), 3681
Schwadron, N. A., Fisk, L. A., \& Gloeckler, G. 1996, Geophys. Res. Lett., 23 2871

Simunac, K. D. C., \& Armstrong, T. P. 2004, J. Geophys. Res., 109, A10101

Stone, E. C., Frandsen, A. M., Mewaldt, R. A., Christian, E. R., Margolies, D., Ormes, J. F., \& Snow, F. 1998, Space Sci. Rev., 86, 1

Stone, E. C., et al. 2005, Science, 309, 2017

Tylka, A. J., et al. 2000, in AIP Conf. Proc. 528, Acceleration and Transport of Energetic Particles Observed in the Heliosphere, ed. R. A. Mewaldt et al. (New York: AIP), 147

von Rosenvinge, T. T., et al. 1995, Space Sci. Rev., 71, 155

von Steiger, R., et al. 2000, J. Geophys. Res., 105, 27217

Webb, G. M., Ko, C. M., Zank, G. P., \& Jokipii, J. R. 2003, ApJ, 595, 195

Wiedenbeck, M. E., et al. 2003, in AIP Conf. Proc. 679, Solar Wind Ten, ed. M. Velli, R. Bruno, \& F. Malara (Melville, NY: AIP), 252

Wiedenbeck, M. E., et al. 2005, in Proc. 29th Int. Cosmic Ray Conf., ed. B. Sripathi Acharya et al. (Mumbai: Tata Institute of Fundamental Research), 117 